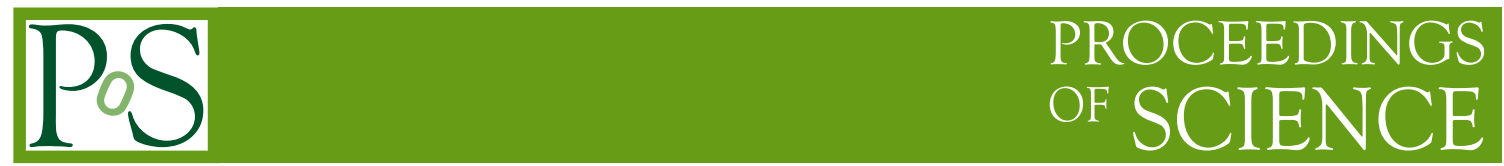

\title{
Top quark physics results with the ATLAS experiment
}

\author{
M. I. Besana*t \\ Università degli Studi di Milano and INFN Milano \\ E-mail: ilaria.besana@mi.infn.it
}

The top quark physics program is one of the milestones of the physics program of the ATLAS experiment at the Large Hadron Collider. This report will focus on the recent results obtained using pp collisions data at $\sqrt{s}=7 \mathrm{TeV}$ collected in 2011. In particular the measurement of top quark pair and single top quark production cross sections will be described, as well as the measurement of top quark main properties. Searches for new physics processes involving top quarks will be finally presented.

LHC on the March,

November 16-18, 2011

Protvino, Moscow region, Russian Federation

\footnotetext{
* Speaker.

${ }^{\dagger}$ For the ATLAS Collaboration
} 


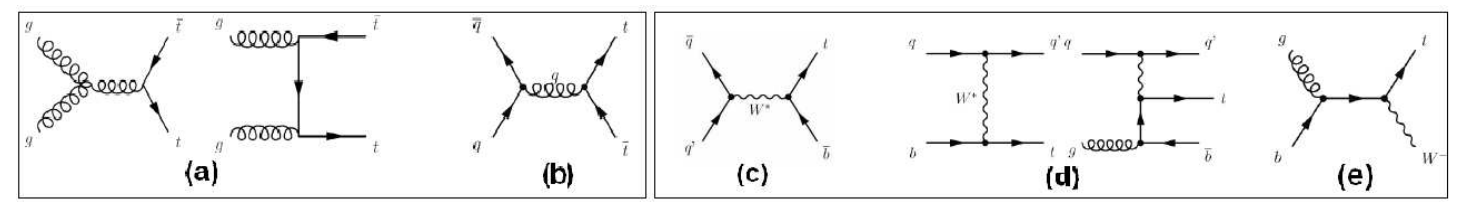

Figure 1: LO production diagrams for $t \bar{t}$, g-g fusion (a) and $q \bar{q}$ annhilation (b), and single top quark: $s$ - (c), $t$ - (d) and $W t$-channel (e).

\section{Introduction}

Top quark physics is presently and will be widely explored at the Large Hadron Collider (LHC) with the ATLAS detector [1]. This physics field is topical and interesting because of the peculiarity of the top quark. Since it is characterized by a very short lifetime, it decays before hadronizing, giving the possibility to study the properties of a bare quark. Top quark events can also provide consistency tests of the Standard Model (SM) and can be used to search for new physics.

Top quarks can be produced in pairs $(t \bar{t})$ and the production cross section in pp collisions at $7 \mathrm{TeV}$ centre-of-mass energy has been calculated at NNLO to be $165_{-16}^{+11} \mathrm{pb}$ assuming a top quark mass of $172.5 \mathrm{GeV}$ [2]. The LO production mechanisms are $g g$-fusion, which gives a contribution of $85 \%$, and $q \bar{q}$-annihilation, as shown in Figure 1. The production of (anti)top quark alone (single top quark) can occur through three different production mechanisms as shown in Figure 1: $t$-, $W t$ and $s$-channel. The cross sections have been calculated at NNLO to be $\sigma_{t-c h}=64.6_{-2.6}^{+3.3} \mathrm{pb}$ [3], $\sigma_{W t-c h .}=15.7 \pm 1.4 \mathrm{pb}[4]$ and $\sigma_{s-c h}=4.6 \pm 0.3 \mathrm{pb}[5]$.

The LHC has been producing collisions at a centre-of-mass energy of $7 \mathrm{TeV}$ since March 2010. The delivered integrated luminosity to the ATLAS experiment is about $5 \mathrm{fb}^{-1}$ and the peak luminosity was of $3.7 \cdot 10^{33} \mathrm{~cm}^{-2} \mathrm{~s}^{-1}$. The results presented here are based on 2011 data and when not stated otherwise they have been produced using data collected until the end of June and corresponding to $0.70 \mathrm{fb}^{-1}$.

\section{Cross section measurements}

\subsection{Top quark pair production measurement}

The ATLAS collaboration has measured the top quark pair production cross section $\left(\sigma_{t \bar{t}}\right)$ at first with 2010 data [6] [7] [8]. The $\sigma_{t \bar{t}}$ has been then measured again with 2011 data: more precise results have been obtained and almost all the decay channels predicted by the SM have been included.

According to the SM top quarks decay almost exclusively into a $W$ boson and a $b$ quark $(t \rightarrow W b)$. The decay modes of top quark pairs are classified according to $W$ bosons decay modes, into lepton + jets channel, where one $W$ boson decays into $l+v$ and the other one into two jets, the dilepton channel, where both $W$ bosons decay into $l+v$, and the all hadronic channel where both $W$ bosons decay into two jets.

\subsubsection{Measurement of inclusive $\sigma_{t \vec{t}}$}

Lepton+jets channel: Signal events have been selected requiring the presence of only one 

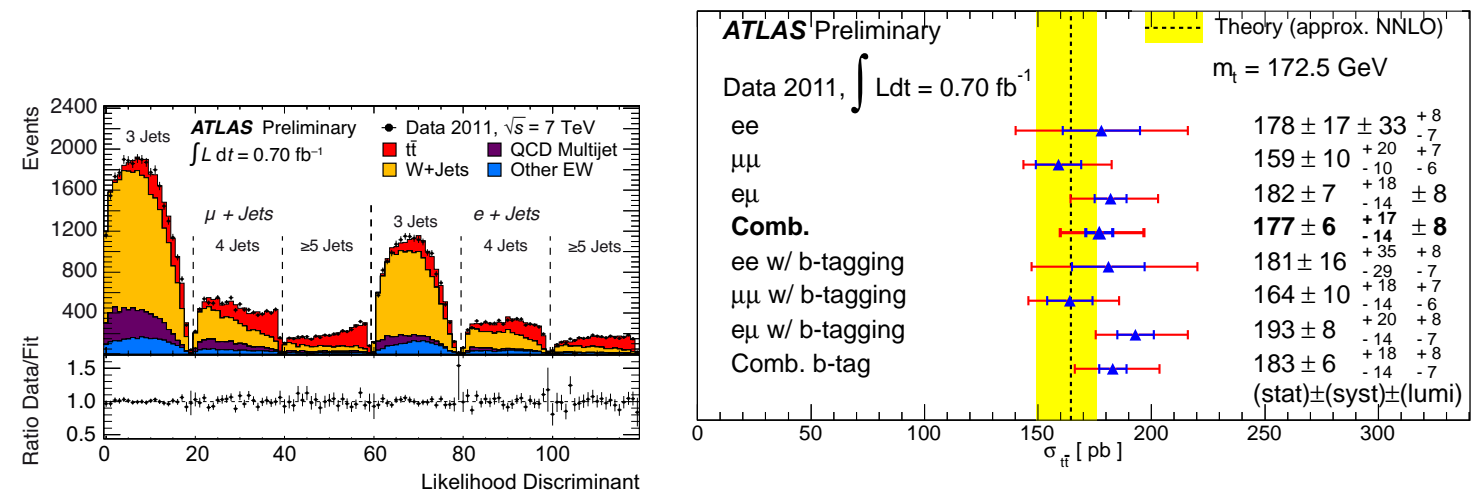

Figure 2: Left plot: Likelihood discriminant in the three-jet bin, the four-jet bin and the five-jet bin of the e+jets and $\mu+$ jets channels [9]. The lower plot shows the ratio of data and the fitted signal and background contributions. Uncertainties in the ratio include data and Monte Carlo statistical uncertainty. Right plot: Cross section summary for individual dilepton channels and for their combination with and without the $b$-tagging request [10].

lepton in the event, either an electron or a muon ${ }^{1}$, and applying a cut on missing transverse momentum $\left(E_{\mathrm{T}}^{\text {miss }}\right)$ and on lepton- $E_{\mathrm{T}}^{\text {miss }}$ transverse mass. The current best $\sigma_{t \bar{t}}$ measurement at $7 \mathrm{TeV}$ has been obtained in this channel without the use of $b$-tagging information [9]. The dominant background contribution arise from $W+$ jets events. The signal has been separated from the background making use of the differences between $t \bar{t}$ and $W+$ jets events. A likelihood discriminant has been built including six different channels: exactly three jets, exactly four jets and at least five jets in e and $\mu$ channel, as shown in the left plot of Figure 2 .

The $\sigma_{t \bar{t}}$ has been extracted from a fit and found to be: $\sigma_{t \bar{t}}=179.0 \pm 3.9$ (stat.) \pm 9.0 (syst.) \pm 6.6(lumi.) $\mathrm{pb}$, where the last term is the contribution coming from luminosity uncertainty. With a total uncertainty of $7 \%$, this measurement exceeds the precision of the theoretical calculation. The most important systematic uncertainties are signal modelling, jet energy scale (JES) uncertainty and muon identification uncertainty.

Dilepton channel: As for the lepton+jets channel, only events with electrons or muons in the final state have been considered. Signal candidate events have been selected requiring the presence of two opposite charge leptons and at least two jets. The background contribution, dominated by QCD and $Z+$ jets events, has been suppressed by requiring large $E_{\mathrm{T}}^{\text {miss }}$ for same flavour events, while for $e \mu$ channel it has been required a large $H_{\mathrm{T}}$, defined as the scalar sum of jet and lepton transverse momenta.

Two complementary measurements with and without the $b$-tagging request [10] have been performed. A counting method has been used to extract the cross section. The two main backgrounds have been estimated from data, while other backgrounds and signal acceptance from Monte Carlo.

Figure 2 (right) summarises the cross sections extracted for all the different channels. A precision of $11 \%$ has been obtained. The result is already limited by systematic uncertainties and the

\footnotetext{
${ }^{1}$ Small contributions to $W \rightarrow l$ from $W \rightarrow \tau \rightarrow l$ are included. Events with an hadronically decaying $\tau$ has been treated separately.
} 
most important contributions come from the uncertainty on luminosity, on jet energy scale and, for the measurement that use $b$-tagging, on $b$-tagging calibration.

All hadronic channel: Top quark pair cross section has been measured in the all hadronic channel using $1.02 \mathrm{fb}^{-1}$ [11]. This measurement is challenging due to the huge QCD multi-jet background contamination.

Events have been selected on line using a multi-jet trigger. Off line selection requires the presence of at least six jets, two of them identified as $b$-jets. A minimal separation between the two $b$-jets has been required. Moreover a cut has been applied on $E_{\mathrm{T}}^{\mathrm{miss}}$ significance ${ }^{2}$. A discriminating variable, $\chi^{2}$, has been built using top quark mass and $W$ boson mass as constraints, for three jets and jet pair invariant masses respectively. The signal fraction has been extracted from a fit on the $\chi^{2}$ distribution. The signal templates have been taken from Monte Carlo, while the one for QCD background has been taken from data, using control regions at low jet multiplicity.

The cross section has been found to be: $\sigma_{t \bar{t}}=167 \pm 18$ (stat.) \pm 78 (syst.) \pm 6 (lumi.) pb. The most important contributions to systematic uncertainty come from Initial and Final State Radiation (ISR and FSR) modelling, JES and $b$-tagging calibration uncertainty.

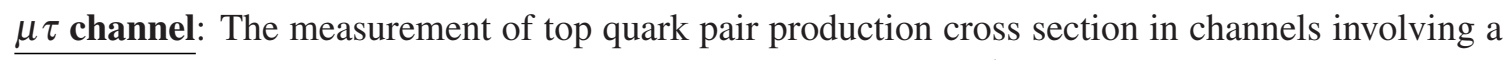
$\tau$ lepton is interesting for searches for a charged Higgs boson $\left(H^{ \pm}\right)$[12]. If the top quark can decay into a $b$ quark and a $H^{ \pm}$boson, branching ratios of final states involving $\tau$ leptons are enhanced. This study has been performed using data corresponding to an integrated luminosity of $1.1 \mathrm{fb}^{-1}$ and considering events with one muon and one hadronically decaying $\tau$. Boosted decisions trees (BDT) have been used to identify $\tau$ leptons and reject jets or electrons. In particular, the signal fraction has been extracted from a fit on the BDT used to separate $\tau$ 's from jets.

The cross section has been found to be: $\sigma_{t \bar{t}}=142 \pm 21$ (stat.) ${ }_{-16}^{+20}$ (syst.) \pm 5 (lumi.) pb. A $20 \%$ precision has been reached. Statistical uncertainty is still significant and the main systematic uncertainty come from $\tau$ - and $b$-jet identification uncertainties and from ISR/FSR modelling.

\subsubsection{Jet multiplicity in top quark pair events}

The aim of this measurement is to provide constraints on ISR at the top quark mass scale [13]. The ISR modelling uncertainty has indeed a large impact on many top quark related results. Jet multiplicity in $t \bar{t}$ events in data has been compared to Monte Carlo predictions, obtained scaling ISR up and down within the scale uncertainty. No discrimination is possible with present uncertainty. The major limitation is background uncertainty at low jet multiplicity and JES uncertainty at high jet multiplicity. The measurement would benefit from more data: the ISR contribution will be cleanly probed by providing more events with high- $p_{\mathrm{T}}$ jets.

\subsubsection{Measurement of $t \bar{t}+\gamma$ cross section}

This measurement has been performed using data corresponding to $1.04 \mathrm{fb}^{-1}$ with the aim of acquiring some knowledge on the $t \gamma$ vertex [14].

Signal events in single lepton channel have been considered. Apart from standard lepton+jets selection cuts (including the request of one tagged jet), the presence of one identified photon has

\footnotetext{
${ }^{2} E_{\mathrm{T}}^{\text {miss }}$ significance is defined as: $E_{\mathrm{T}}^{\text {miss }} / \sqrt{H_{\mathrm{T}}}$, where $H_{\mathrm{T}}$ is the scalar sum of the transverse momentum of all jets in the event.
} 

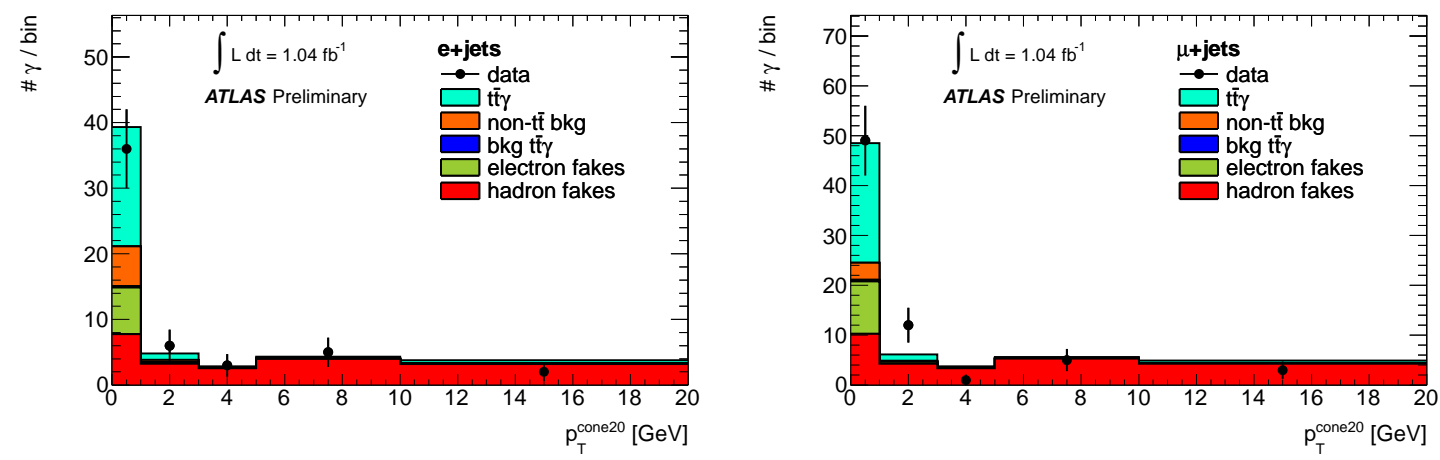

Figure 3: Result of the template fit in the electron (left) and muon channel (right) [14]. The signal is shown on top of background contributions.

been required. The most important backgrounds come from $t \bar{t}$ events + one hadron or one electron misidentified as a photon and non- $t \bar{t}$ events ( $W+$ jets, $Q C D, Z+$ jets, single top quark and dibosons) produced in association with a photon or a fake electron. All backgrounds, apart from the small contributions from $Z+$ jets, single top quark and dibosons, have been estimated from data, using measurements in control regions. The contribution of $t \bar{t}+\gamma$ events, that don't satisfy acceptance selection cuts have been estimated as well.

The number of signal events have been extracted from a fit on photon isolation distribution, shown in Figure 3. The cross section has been finally extracted using the acceptance estimated from Monte Carlo and found to be $\sigma_{t \bar{t}+\gamma}=2.0 \pm 0.5$ (stat.) \pm 0.7 (syst.) \pm 0.08 (lumi.) pb. This measurement is already limited by systematic uncertainties and the main contributions come from $\gamma$ identification efficiency and purity, JES uncertainty and ISR/FSR modelling. Higher statistics will help in reducing systematic uncertainty, especially the contribution related to $\gamma$ identification.

\subsection{Single top quark production cross section}

$t$-channel: The single top cross section has been measured in this channel, considering only events with the $W$ boson decaying into one electron or one muon [15]. The cross section has been obtained from a counting method. Signal events have been selected using $b$-tagging and applying optimized cuts, as for example on lepton- $E_{\mathrm{T}}^{\text {miss }}$ transverse mass. The main backgrounds are $t \bar{t}, W+\mathrm{jets}$ and QCD. The first one has been obtained from Monte Carlo, while the other two from data.

The cross section has been measured to be $\sigma_{t-c h}=90 \pm 9$ (stat.) ${ }_{-20}^{+31}$ (syst.) pb, in agreement with the SM prediction. The main systematic uncertainties arise from ISR/FSR modelling and $b$-tagging uncertainty.

Wt-channel: The single top quark cross section has been measured in the $W t$-channel using a counting method, selecting events with both $W$ bosons decaying leptonically [16]. Selection cuts have been optimized to reject the main backgrounds: $Z \rightarrow e e, \mu \mu, \tau \tau$ and $t \bar{t}$. Residual background contributions have been estimated using control samples in data.

The signal cross section has been found to be $\sigma_{W t-c h}=14_{-5.1}^{+5.3}$ (stat. ${ }_{-9.4}^{+9.7}$ (syst.) pb, in agreement with the SM prediction. The statistical uncertainty is still important; the main systematic uncertainties arise from uncertainties on JES, jet energy resolution (JER), jet reconstruction efficiency and $t \bar{t}$ contamination. 


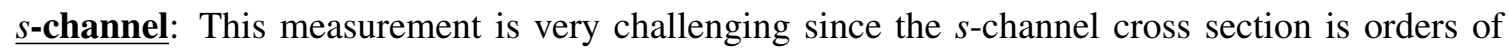
magnitude lower than the one of the most important backgrounds ( $t \bar{t}$ and $W+$ jets). At the day of writing an upper limit at $26.5 \mathrm{pb}$ has been obtained in this channel [17].

\section{Top quark properties}

Top quark mass: The measurement of top quark mass is an important goal of the LHC, since it provides constraints on that of the Higgs boson.

The ATLAS measurement has been performed using a template method [18]. Signal events have been selected in the lepton+jets channel, requiring the presence of one $b$-jet. Top quark mass has been reconstructed considering the three jets with the highest $p_{\mathrm{T}}$ sum. The obtained distribution in data has been compared to signal+background templates, created with different values of top quark quark mass and JES. Both quantities have been then extracted from a simultaneous fit, in order to reduce JES impact on top mass quark measurement.

The obtained mass value is $m_{t o p}=175.9 \pm 0.9$ (stat.) \pm 2.7 (sys.) GeV. The result is already limited by systematic uncertainty and the main contributions come from signal modelling and JES uncertainty.

Top quark charge asymmetry: In the Standard Model, a top quark charge asymmetry only occurs via asymmetric initial states in $t \bar{t}$ production, therefore the process $q \bar{q} \rightarrow t \bar{t}$ gives the main contribution to the asymmetry. This asymmetry comes from the fact that top quarks are emitted preferentially in the direction of the incoming quark and not in the one of the incoming antiquark. The LHC is a proton-proton collider and this asymmetry manifests itself in the fact that top quarks will be more boosted than antitops and so top quarks will be characterized by a broader rapidity distribution. A sensible observables to the asymmetry has been therefore defined as:

$$
A_{C}=\frac{N(\Delta|Y|>0)-N(\Delta|Y|<0)}{N(\Delta|Y|>0)+N(\Delta|Y|<0)}
$$

where $\Delta|Y|=\left|Y_{t}\right|-\left|Y_{\bar{t}}\right|, Y_{t(\bar{t})}$ is top (antitop) quark rapidity, $N(\Delta|Y|>0$ ) is the number of events in which $\Delta|Y|$ is positive and $N(\Delta|Y|<0)$ is the number of events in which $\Delta|Y|$ is negative. In the SM the asymmetry is expected to be small, 0.6\%, according to MC@ NLO predictions. Some BSM theories predict, at opposite, significantly larger asymmetries.

This measurement has been performed in lepton+jets channel, using $b$-tagging information [19]. The two main backgrounds, $W+$ jets and QCD, have been estimated from data and top quark kinematics has been reconstructed using a kinematic fit. A raw asymmetry has been then extracted from background subtracted $|\Delta Y|$ distribution and the obtained result has been finally corrected for acceptance and detector effects applying an unfolding technique. The parton level asymmetry has been found to be $A_{C}=-2.4 \pm 1.6$ (stat.) \pm 2.3 (syst.)\%, in agreement with the SM prediction. This result is limited by systematic uncertainty and the main contributions are signal modelling and JER uncertainty.

$\underline{W t b \text { vertex: }}$ The $W$ boson helicity has been measured using a template method to check the V-A structure of the $W t b$ vertex. The measurement has been performed both in lepton+jets and dilepton channel and the observable used is the angle, $\cos \theta^{*}$, between the lepton and the inverse of the momentum direction of the $b$ quark in the $W$ boson rest frame [20]. The $\cos \theta^{*}$ distribution has 
been fitted to extract the helicity fractions, which has been found to be in agreement with the SM prediction. The main systematic uncertainties arise from signal modelling and $b$-jet identification uncertainty.

Spin correlations: The measurement of top quark pair spin correlation at the LHC is a test of the SM complementary with respect to the previous Tevatron results, due to the different LHC dominant production mechanism ( $g g$-fusion). The angular distribution of top quark decay products has been studied [21]. The measurement has been performed in the dilepton channel and the chosen observable is the angle between the two charged leptons in the laboratory frame $\Delta \Phi\left(l^{+}, l^{-}\right)$. This variable is really interesting, since it doesn't need the reconstruction of event kinematics. The $\Delta \Phi\left(l^{+}, l^{-}\right)$distribution in data has been fitted with the SM and the no-correlation predictions. The SM contribution has been found to be $f_{S M}=1.06 \pm 0.21$, combining all lepton pairs channels. The most important systematic uncertainties come from Monte Carlo available statistics and signal modelling.

Top quark charge: The top quark charge has been measured to exclude the hypothesis of an exotic top quark with a charge of $-\frac{4}{3}$ [22].

Signal events have been selected in the lepton+jets channel, requiring the presence of at least one $b$-jet. Top quark charge can be measured from the one of its decay products, the $W$ boson and the $b$ quark. Two crucial points of this measurement are therefore a correct pairing of $W$ boson and $b$-jet and the measurement of their charges. $W$ boson charge can be measured from the one of the lepton, while for $b$-jet charge measurement, two techniques have been used. The first one uses the sum of the charges of the tracks associated to the $b$-jet, while the second one takes advantage from the fact that there is a correlation between muons charge and $b$ hadrons charge, in $b$ hadrons semileptonic decays. In the second case, only events with a soft muon inside a $b$-jet have been considered and the $b$ quark charge has been determined from the muon one.

Good agreement with the SM has been observed: an exotic top quark has been excluded with more than $5 \sigma$ with both techniques, combining electron and muon channels.

FCNC: Flavour changing neutral currents (FCNC) are highly suppressed according to the SM, but they can be enhanced in some BSM scenarios.

The ATLAS collaboration has recently searched for a top quark decaying into a $Z$ boson and a quark [23]. This study has been performed looking for $t \bar{t}$ events with one top quark decaying into a $W$ boson and a $b$-jet and the other one through FCNC into a $Z$ boson and one quark. Both $W$ and $Z$ bosons have been required to decay leptonically. The presence of three leptons, two of them with same flavour and opposite charge, in the final state is very powerful in order to minimise the amount of the background. The most important contributions come from dibosons events which have has been estimated from Monte Carlo. Other backgrounds, such as $Z+$ jets, $W+$ jets, $t \bar{t}$ and QCD, characterized by fake leptons have been estimated from data. No signal evidence have been observed as can be seen from Figure 4 and an upper limit has been obtained: $B R(t \rightarrow q Z)<1.1 \%$ at $95 \%$ of CL.

\section{Search for new physics in $t \bar{t}$ events}

Some BSM theories predict the existence of new resonances that decay predominantly into top quark pairs. 

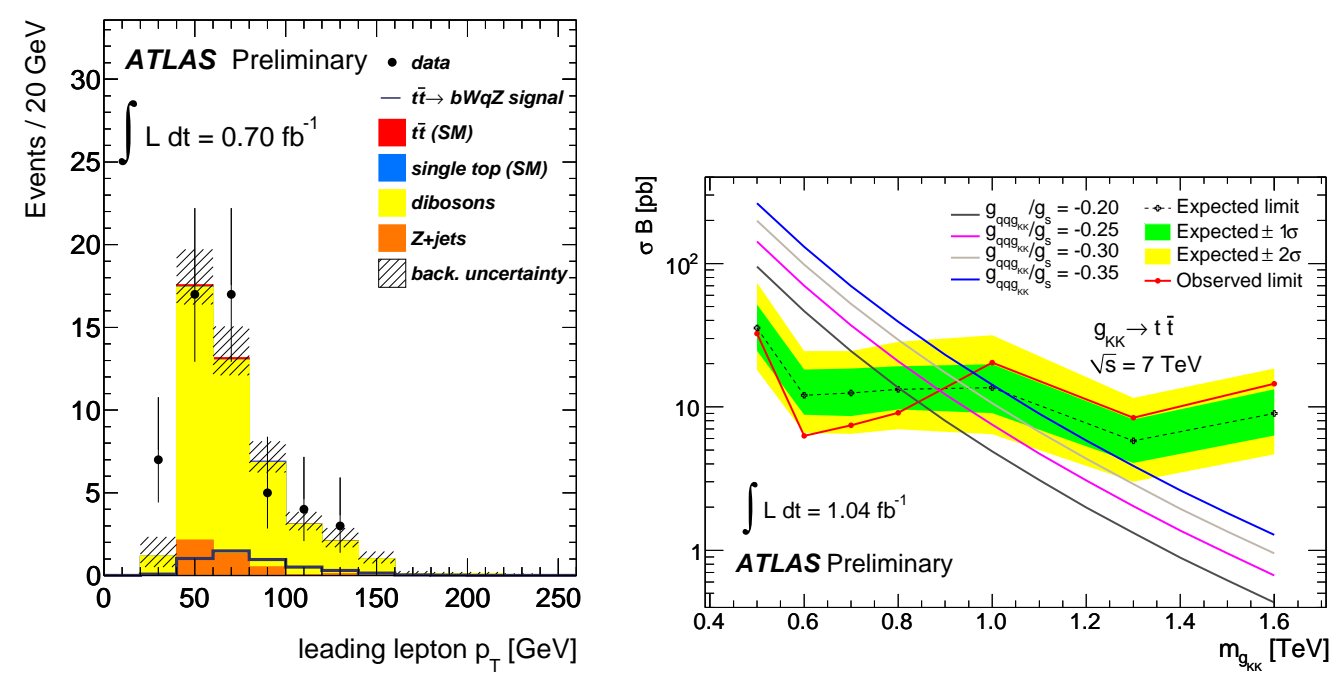

Figure 4: Left plot: Distribution of the transverse momentum of the leading lepton [23]. The background uncertainties shown include the Monte Carlo simulation statistical uncertainties and the DD uncertainties. The signal distributions are normalized to the observed BR limit, at 95\% CL. Right plot: Expected and observed limits at 95\% C.L. on cross section times BR and expected cross section for a Randall-Sundrum KK-gluon [24].

A search in the dilepton channel has been performed using data corresponding to an integrated luminosity of $1.04 \mathrm{fb}^{-1}$ [24]. In particular the $H_{T}+E_{\mathrm{T}}^{\text {miss }}$ distribution has been studied to look for an excess. The production of a Kaluza Klein-gluon in Randall-Sundrum model has been investigated. The main SM backgrounds are Drell-Yan+jets, QCD, $t \bar{t}$, single top quark and dibosons. The first two have been estimated using data, while the others have been taken from Monte Carlo predictions. Data have been found to be compatible with the SM background only hypothesis. Upper limits at the $95 \% \mathrm{CL}$. have been set on the cross section times the branching ratio (BR) of the resonance decaying into $t \bar{t}$ as a function of the resonance mass, as shown in Figure 4. A lower mass limit has been set on KK-gluon mass at $0.84 \mathrm{TeV}$ at $95 \% \mathrm{CL}$. The main systematic uncertainties are JES and $t \bar{t}$ modelling.

Furthermore a search for heavy resonances in the $t \bar{t}$ invariant mass spectrum has been performed in lepton+jets channel with $200 \mathrm{pb}^{-1}$ [25]. No signal evidence has been observed and limits at $95 \%$ C.L have been set for the production cross section of a $Z$ ' boson, multiplied for the decay BR into top quark pairs: $38 \mathrm{pb}$ for $m_{Z^{\prime}}=500 \mathrm{GeV}$ and $3.2 \mathrm{pb}$ for $m_{Z^{\prime}}=1300 \mathrm{GeV}$.

\section{Conclusions}

The ATLAS collaboration has performed a very competitive and wide ranging program in the field of top quark physics. The cross section of the inclusive pair production of top quarks has been measured in almost all the final states predicted by the SM, reaching an accuracy even better than that of theoretical predictions. The measurement of jet multiplicity has been performed to constrain ISR uncertainty and the $t \bar{t}+\gamma$ cross section has been measured for the first time at the LHC. The single top quark production cross section has been measured in $t$ - and $W t$-channel, while 
more data are needed for the $s$-channel. Arguably, the most important top quark properties have been successfully measured: top quark mass, charge, $t \bar{t}$ charge asymmetry, $W$ boson helicity and $t \bar{t}$ spin correlation. All results obtained are consistent with SM expectations and most of them are already limited by systematic uncertainties. Thanks to the rapidly increasing LHC luminosity and a centre-of-mass energy significantly higher with respect to previous colliders, ATLAS has obtained new stringent limits in the searches of new physics in top quark events.

Future measurements will take advantage of using more data. In many cases the use of larger statistics will help also in reducing systematic uncertainties.

\section{References}

[1] ATLAS Collab., JINST 3, S08003 (2008).

[2] S. Moch and P. Uwer, Phys. Rev. D 78, 034003 (2008).

[3] N. Kidonakis, Phys. Rev. D 83, 091503 (2011).

[4] N. Kidonakis, Phys. Rev. D 82, 054018 (2010).

[5] N. Kidonakis, Phys. Rev. D 81, 054028 (2010).

[6] ATLAS Collab., Phys. Lett. B 707, 459-477 (2012).

[7] ATLAS Collab., Eur. Phys. J. C 71, 1577 (2011).

[8] ATLAS Collab., Phys. Lett. B 707, 459 (2012).

[9] ATLAS Collab., Top Quark Pair Production Cross-section Measurements in ATLAS in the Single Lepton+Jets Channel without b-tagging, ATLAS-CONF-2011-023, CERN, Geneva, 2011; https://cdsweb.cern.ch/record/1336753

[10] ATLAS Collab., Measurement of the top quark pair production cross section in pp collisions at $\sqrt{s}=7$ TeV in dilepton final states with ATLAS, ATLAS-CONF-2011-100, CERN, Geneva, 2011; https://cdsweb.cern.ch/record/1369215

[11] ATLAS Collab., Measurement of $t \bar{t}$ production in the all-hadronic channel in $1.02 \mathrm{fb}^{-1}$ of $p p$ collisions at $\sqrt{s}=7 \mathrm{TeV}$ with the ATLAS detector, ATLAS-CONF-2011-140, CERN, Geneva, 2011; https://cdsweb.cern.ch/record/1385033

[12] ATLAS Collab., Measurement of the top quark pair production cross section in pp collisions at $\sqrt{s}=7 \mathrm{TeV}$ in $\mu+\tau$ final states with ATLAS, ATLAS-CONF-2011-119, CERN, Geneva, 2011; https://cdsweb.cern.ch/record/1376411

[13] ATLAS Collab., Reconstructed jet multiplicities from the top-quark pair decays and associated jets in pp collisions at $\sqrt{s}=7 \mathrm{TeV}$ measured with the ATLAS detector at the LHC, ATLAS-CONF-2011-142, CERN, Geneva, 2011; https://cdsweb.cern.ch/record/1385518

[14] ATLAS Collab., Measurement of the inclusive t tbar gamma cross section with the ATLAS detector, ATLAS-CONF-2011-153, CERN, Geneva, 2011; https://cdsweb.cern.ch/record/1398197

[15] ATLAS Collab., Measurement of the t-channel Single Top-Quark Production Cross Section in $0.70 \mathrm{fb}^{-} 1$ of pp Collisions at $\sqrt{\mathrm{s}}=7 \mathrm{TeV}$ with the ATLAS detector, ATLAS-CONF-2011-101, CERN, Geneva, 2011; https://cdsweb.cern.ch/record/1369217

[16] ATLAS Collab., Search for $W+t$ single-top events in the dileptonic channel at ATLAS, ATLAS-CONF-2011-104, CERN, Geneva, 2011; https://cdsweb.cern.ch/record/1369829 
[17] ATLAS Collab., Search for s-Channel Single Top-Quark Production in pp Collisions at $\sqrt{s}=7 \mathrm{TeV}$, ATLAS-CONF-2011-118, CERN, Geneva, 2011; https://cdsweb.cern.ch/record/1376410

[18] ATLAS Collab., Measurement of the top quark mass from 2011 ATLAS data using the template method, ATLAS-CONF-2011-120, CERN, Geneva, 2011; https://cdsweb.cern.ch/record/1376412

[19] ATLAS Collab., Measurement of the charge asymmetry in top quark pair production in pp collisions at $\sqrt{s}=7 \mathrm{TeV}$ using the ATLAS detector, ATLAS-CONF-2011-106, CERN, Geneva, 2011; https://cdsweb.cern.ch/record/1372916

[20] ATLAS Collab., Measurement of the W boson polarisation in top quark decays in $0.70 \mathrm{fb}^{-1}$ of pp collisions at $\sqrt{s}=7 \mathrm{TeV}$ with the ATLAS detector, ATLAS-CONF-2011-122, CERN, Geneva, 2011; https://cdsweb.cern.ch/record/1376422

[21] ATLAS Collab., Measurement of the charge asymmetry in top quark pair production in pp collisions at $\sqrt{s}=7 \mathrm{TeV}$ using the ATLAS detector, ATLAS-CONF-2011-117, CERN, Geneva, 2011; https://cdsweb.cern.ch/record/1372916

[22] ATLAS Collab., Measurement of the top quark charge in pp collisions at $\sqrt{s}=7$ TeV in the ATLAS experiment, ATLAS-CONF-2011-141, CERN, Geneva, 2011; https://cdsweb.cern.ch/record/1385517

[23] ATLAS Collab., A search for Flavour Changing Neutral Currents in Top Quark Decays $t \rightarrow q Z$ at $\sqrt{s}=7 \mathrm{TeV}$ in $0.70 \mathrm{fb}^{-1}$ of pp collision data collected with the ATLAS Detector, ATLAS-CONF-2011-154, CERN, Geneva, 2011; https://cdsweb.cern.ch/record/1398198

[24] ATLAS Collab., A Search for ttbar Resonances in the Dilepton Channel in $1.04 \mathrm{fb}^{-1}$ of pp Collisions at $\sqrt{s}=7 \mathrm{TeV}$, ATLAS-CONF-2011-123, CERN, Geneva, 2011; https://cdsweb.cern.ch/record/1376423

[25] ATLAS Collab., A Search for $t \bar{t}$ Resonances in the Lepton Plus Jets Channel in $200 \mathrm{pb}^{-1}$ of pp Collisions at $\sqrt{s}=7 \mathrm{TeV}$, ATLAS-CONF-2011-087, CERN, Geneva, 2011;

https://cdsweb.cern.ch/record/1356196 